New Generation Computing, 20(2002)215-216

Ohmsha, Ltd. and Springer-Verlag

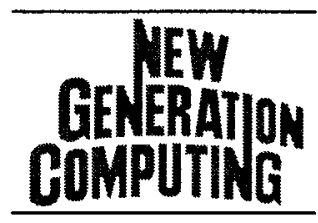

(C)Ohmsha, Ltd. 2002

Preface

\title{
Biomolecular Computing
}

\author{
Masayuki YAMAMURA \\ Interdisciplinary Graduate School of Science and Engineering, \\ Tokyo Institute of Technology \\ 4259 Nagatsuta, Midori-ku, Yokohama 226-8502 \\ CREST, Japan Science and Technology Corporation (JST) \\ my@dis.titech.ac.jp \\ Tom HEAD \\ Mathematical Sciences, Binghamton University \\ Binghamton, New York 13902-6000 \\ tom@math . binghamton. edu \\ Masami HAGIYA \\ Graduate School of Information Science and Technology, \\ University of Tokyo \\ 7-3-1 Hongo, Bunkyo-ku, Tokyo 113-0033 \\ CREST, JST \\ hagiya@is.s.u-tokyo.ac.jp
}

This special issue of New Generation Computing consists of two invited papers and five fully reviewed papers. The contributions in this special issue represent state-of-the-art research ranging from pure theoretical to advanced experimental works. As a research field, Biomolecular Computing is not yet established and is characterized by interdisciplinary worldwide collaboration. We invited three influential project leaders to survey this field from their own viewpoint. Masami Hagiya (the University of Tokyo) has already provided an invited paper for vol. 17, no. 2, pp. 131-140, 1999. John Reif (Duke University) and Grezgorz Rosenberg (Leiden University) have written new papers for this volume.

We placed invited papers first. Other contributions are arranged arbitrarily. Here is a brief summary of the contributions:

- John H. Reif; The Emergence of the Discipline of Biomolecular Computation in the US. Surveys the recent evolution in the US of an emerging technology known as Biomolecular Computation from its early stages to its development and extension into other areas such as nanotechnology.

- Grzegorz Rosenberg; Models of Molecular Computing based on Molecular Reactions. Gives a new perspective with two models of molecular computing, forbidding-enforcing systems and molecular landscape systems. 
- Masahito Yamamoto, Atsushi Kameda, Nobuo Matsuura, Toshikazu Shiba, Yumi Kawazoe and Azuma Ohuchi; A Separation Method for DNA Computing based on Concentration Control. Examines a method to separate and quantify target DNA and shows an experimental application to the shortest path problem.

- Masanori Arita and Satoshi Kobayashi; DNA Sequence Design Using Templates. Introduces a simple strategy that systematically generates a set of sequences such that any of its members will have certain mismatches with other sequences, their compliments and soncatenations.

- Rudolf Freund, Carlos Martín-Vide and Victor Mitrana; On Some Operations on Strings Suggested by Gene Assembly in Ciliates. Defines three operations on strings and languages suggested by the process of gene assembly in hypotrichous ciliates and is devoted to some computational aspects of these operations from a formal language point of view.

- Andrei Păun and Gheorghe Păun; The Power of Communication: P Systems with Symport / Antiport. Considers the biological phenomenon of transmembrane transport of chemicals as the computation by communication, and shows that membrane systems, without changing the used objects, are computationally complete.

- Julia Khodor and David K. Gifford; Experimental Efficiency of Programmed Mutagenesis. Reports the experimental efficiency of primitive operations of computation by programmed mutagenesis on DNA molecules proposed by the authors.

We have a special notice that "DNA Sequence Design Using Templates" by $M$. Arita and S. Kobayashi has been selected as a distinguished paper by the editorial board of the journal and guest editors of the special issue. Sequence design is one of the hardest problems in Biomolecular Computing since the earliest days. Their work provides a real breakthrough for the design of homogeneous general code sets with an elegant two level problem decomposition.

We would like to thank all authors, the external reviewers, and the journal editors for providing their expertise and excellent contributions. 